FORMATION Formation emploi

Revue française de sciences sociales

106 | avril-juin 2009

Pêle-mêle

\title{
Les diplômes transversaux peinent à s'imposer sur le marché du travail
}

Transversale Ausbildungsabschlüsse setzen sich auf dem Arbeitsmarkt nur schwer durch

Cross-disciplinary qualifications struggle for acceptance in the labour market Los diplomas transversales tienen dificultades para imponerse en el mercado de trabajo

\section{Emmanuel Quenson}

\section{OpenEdition}

\section{Journals}

Édition électronique

URL : http://journals.openedition.org/formationemploi/1908

DOI : 10.4000/formationemploi.1908

ISSN : 2107-0946

Éditeur

La Documentation française

Édition imprimée

Date de publication : 1 juin 2009

Pagination : 25-39

ISSN : 0759-6340

Référence électronique

Emmanuel Quenson, «Les diplômes transversaux peinent à s'imposer sur le marché du travail », Formation emploi [En ligne], 106 | avril-juin 2009, mis en ligne le 01 mars 2011, consulté le 30 octobre 2020. URL : http://journals.openedition.org/formationemploi/1908 ; DOI : https://doi.org/10.4000/ formationemploi. 1908 


\title{
Formation
}

\section{Les diplômes transversaux peinent à s'imposer sur le marché du travail'}

\author{
Emmanuel Quenson*
}

\author{
Les diplômes transversaux sont censés s'ajuster à la flexibilité \\ des organisations et à la recomposition des métiers dans plusieurs branches. \\ Pourtant, l'exemple d'un baccalauréat professionnel montre \\ que la transversalité peine à s'imposer sur le marché du travail.
}

Depuis vingt-cinq ans, plusieurs réformes ont renouvelé les diplômes, les cursus et les objectifs de l'enseignement professionnel en fonction de la double nécessité de transmettre des connaissances et une culture pour tous, et de répondre aux nouvelles exigences du marché du travail. Une des mesures phare de cette politique a été la création du baccalauréat professionnel en 1985. Le développement de l'alternance école-production dans tous les segments de l'enseignement professionnel a contribué à rapprocher les sphères autonomes que sont l'école et l'entreprise. Ce modèle pédagogique a revalorisé le rôle de l'entreprise en matière de formation. La consultation obligatoire des représentants des entreprises et des salariés dans les CPC (commissions professionnelles consultatives) représente aussi une

\footnotetext{
${ }^{1}$ Cet article provient de plusieurs rapports financés par la DESCO (Direction de l'Enseignement scolaire - ministère de l'Éducation nationale) consacrés aux diplômes spécialisés et aux diplômes transversaux. Il a bénéficié, dans ses versions successives, des lectures critiques de Lucie Tanguy (université Paris Ouest Nanterre La Défense) et Fabienne Maillard (DESCO). Françoise Ropé (université d'Amiens) m'a fourni des informations qui m'ont aidé à traiter certaines questions. Je tiens à les remercier toutes très sincèrement
}

* Emmanuel Quenson est sociologue, maître de conférences à l'université d'Evry-Val-d'Essonne et chercheur au CPN (Centre Pierre Naville) et à TEPP (Travail, Emploi, Politiques Publiques, FR - Fédération de recherches $\left.n^{\circ} 3126, C N R S\right)$. II étudie les relations entre la formation (initiale et continue) et la gestion de l'emploi et de la maind'œuvre dans l'industrie et les services. Ses recherches les plus récentes portent sur l'émergence de la formation professionnelle continue, les politiques de formation et d'emploi dans les grandes entreprises et la conception des diplômes. Il a notamment publié : Quenson E. (2007), « Informer pour faire adhérer (1971-1976) 》, in Brucy G., Caillaud P., Quenson E., Tanguy L., Former pour réformer. Retour sur la formation permanente (1945-2004), La Découverte, Paris ; Moutet A., Quenson E. (2007), " Formation professionnelle et formation technique au cours des Trente Glorieuses », in Costa-Lascoux J., Dreyfus-Armand G., Témime E. (Dir.), Renault sur Seine. Hommes et lieux de mémoires de l'industrie automobile, La Découverte, Paris ; Quenson E. (2008), "Les diplômes transversaux améliorentils la correspondance entre la formation et l'emploi ? », in Maillard F (Dir.), Des diplômes aux certifications professionnelles? Nou velles normes et nouveaux enjeux, Presses Universitaires de Rennes, Rennes, coll. "Des sociétés ». 
décision significative de cette politique, puisqu'elle a permis aux acteurs du monde professionnel de s'exprimer lors de toute création ou rénovation de diplômes.

Parmi ces évolutions, qui ont toutes pour point commun de mettre les préoccupations de l'emploi au sein du système éducatif, l'une d'entre elles est restée jusqu'alors dans l'ombre, connue seulement de quelques experts de la politique éducative et de certains représentants des branches. Elle concerne le développement d'une nouvelle génération de diplômes appelés communément «diplômes transversaux ». Apparus au milieu des années 80, en premier lieu dans certains BTS (brevet de technicien supérieur) des spécialités tertiaires (Quenson, 2001) et des bacs pro de la maintenance (Fourcade, 1997), ils ont depuis été étendus aux diplômes de la production. À la différence des diplômes spécialisés, dont l'objectif est de former pour des branches bien identifiées, ils préparent à l'emploi dans plusieurs branches. À cette transversalité liée au secteur s'ajoute une transversalité entre fonctions. Elle revient à considérer que la recomposition des frontières entre métiers s'accélère pour donner naissance à des activités professionnelles transversales qui étaient antérieurement divisées. Ces diplômes marquent donc une rupture par rapport à la méthode antérieure de fabrication des diplômes de la production qui les faisait correspondre à des fonctions et des secteurs déterminés.

Ce changement de la politique des diplômes s'inscrit aussi dans le développement d'un nouveau modèle pédagogique exprimé en termes de compétences. Pour répondre aux critiques récurrentes sur le caractère inadapté des diplômes, les responsables de l'Éducation nationale ont permis aux représentants des organisations d'employeurs d'exposer leurs attentes en compétences professionnelles dans les différentes fonctions de l'organisation du travail en les renseignant par une terminologie définie et selon un classement méthodique dans les référentiels des activités professionnelles et de certification. Ces référentiels sont devenus les outils dédiés à la communication entre les différents acteurs qui instruisent l'élaboration des diplômes. Ils attestent aussi que le pilotage des enseignements s'opère de plus en plus par l'aval.
Alors que la plupart des recherches s'intéressent à la formation comme condition première d'accès à l'emploi, nous examinerons les rapports sociaux, souvent occultés, qui président à cette relation entre titres et places dans l'organisation du travail. C'est ainsi que nous verrons, dans une première partie, comment, lors de la création d'un baccalauréat professionnel (Quenson, 2004) préparant aux fonctions de «technicien de production automatisée", certains représentants des professionnels - en l'occurrence ceux de la métallurgie - et l'administration du ministère de l'Éducation nationale se sont entendus pour faire des diplômes transversaux une réponse plus appropriée que les diplômes spécialisés, face au développement supposé des compétences transversales dans les entreprises.

Puis nous constaterons, dans une deuxième partie, que le consensus entre experts du système éducatif et des branches professionnelles se heurte bien souvent à la réalité des entreprises, notamment à l'occasion des périodes de formation en entreprise organisées au cours des études. En effet, un grand nombre de savoirs indispensables à l'obtention du titre dépend des équipements de production et des organisations du travail. Or nous observons un décalage important entre les objectifs du diplôme, qui appréhendent les activités professionnelles comme un ensemble fini d'éléments descriptibles relativement étendus, et la réalité des organisations de la production qui attribuent aux jeunes des tâches bien plus restreintes.

Cette situation n'est pas sans conséquence sur l'insertion professionnelle. La relation d'équivalence entre la formation et la qualification, établie au cours de la fabrication du diplôme, se retrouve rarement dans les entreprises. Dans la troisième partie, nous verrons que la mise au travail s'effectue le plus souvent dans des emplois ouvriers, alors que les perspectives initiales situaient les jeunes au niveau technicien. Si une telle situation révèle les processus sélectifs de recrutement dont sont victimes les débutants (Couppié, Giret, Lopez, 2005), elle est surtout le signe que les experts en charge des diplômes ont souvent trop tendance à anticiper et à généraliser les changements de la distribution des activités professionnelles et des qualités de la main-d'œuvre, sur la base de constats qui ne constituent qu'une déclinaison de la réalité parmi d'autres. 


\section{Encadré 1 \\ Méthodologie}

Le matériau de cette recherche est constitué des comptes-rendus des séances plénières de la 3e commission professionnelle consultative "métallurgie, mécanique, électrotechnique et électronique », et d'une quarantaine d'entretiens semi-directifs d'une durée moyenne d'une heure et demie réalisés entre 2001 et 2004 avec les représentants des branches professionnelles, chefs d'établissement, chefs de travaux, professeurs des matières générales, techniques et professionnelles des lycées professionnels, des Centres de formation d'apprentissage industriel (CFAll, et avec les responsables du personnel et de la production d'entreprises de plusieurs régions (Bourgogne, Bretagne, lle-de-France, Midi-Pyrénées, Nord, Picardie). Les thèmes abordés ont concerné la création du diplôme, sa mise en œuvre dans les établissements, l'organisation de la période de formation en entreprise, le recrutement, les premières années de vie professionnelle des jeunes et leurs activités professionnelles. Une dizaine d'entretiens a aussi été effectuée avec des jeunes en cours de formation. De la sorte, les actions et les représentations de l'ensemble des catégories d'acteurs entrant en jeu dans la définition de la situation sociale observée ont été traitées selon la méthode de l'analyse thématique. Elle a consisté à déterminer des thèmes principaux pour rendre compte du contenu des entretiens, puis à définir des thèmes plus secondaires pour spécifier les différents aspects des premiers.

Les régions enquêtées ont été choisies pour leur représentativité dans l'offre de formation et la diversité des modes de préparation (voie scolaire, apprentissage, formation professionnelle continue). Toutes les entreprises enquêtées ont accueilli des jeunes en alternance. Certaines ont procédé à des recrutements depuis six ans environ. Leurs coordonnées nous ont été fournies par les établissements. Ces entreprises ont été sélectionnées en fonction de critères déterminants pour mesurer la situation des jeunes : secteurs industriels (agro-alimentaire, automobile, chimie, cosmétiques, imprimerie, informatique, mécanique, papiers et carton, plasturgie, routage), tailles (TPE, PMI, grandes entreprises) et positions dans l'organisation industrielle (donneurs d'ordre, sous-traitants).

\section{LA FABRICATION D'UN DIPLÔME TRANSVERSAL}

En France, la fabrication des diplômes est la marque de l'action volontariste de l'État en matière de régulation des relations entre la formation et l'emploi (Verdier, 1995). Le travail en CPC sur l'offre de diplômes est généralement plus politique que technique (Maillard, 2005b). Ainsi, plusieurs travaux ont déjà souligné la prévalence du Secrétariat général des CPC par rapport aux organisations professionnelles et syndicales dont le rôle est second (Fourcade, 1997)2. Il arrive aussi que la création des diplômes se

\footnotetext{
${ }^{2}$ L'initiative de l'administration a été déterminante pour la création des bacs pro «Maintenance des systèmes de mécaniques automatisés » (MSMA) et "Équipements et installations électriques » (EIE).
}

décide avant la fin de la procédure de consultation des partenaires (Pillet, 1995). La consultation des $\mathrm{CPC}$ se mue alors en simple information. De puissantes fédérations professionnelles peuvent être aussi à l'initiative de la création ou de la réforme des diplômes. Mais le recours à l'expertise pour apprécier l'intérêt de cette demande ne donne pas toujours lieu à débat (Ourtau, 1995). L'administration, en dernier ressort, décide de donner une suite favorable ou d'abandonner le projet. De même, le rôle des assemblées plénières est souvent diminué par le travail des groupes de travail dont l'appartenance institutionnelle ne recouvre pas celle des membres des CPC.

La construction du diplôme qui nous intéresse ne remet pas en cause ces constats. Là aussi, l'administration a contrôlé de bout en bout la procédure, une 


\section{Encadré 2}

\section{L'organisation du travail des Commissions professionnelles consultatives}

Créées en 1972, les CPC (Commissions professionnelles consultatives) sont un dispositif paritaire de consultation obligatoire composé de quatre collèges (employeurs, salariés, pouvoirs publics et personnalités qualifiées) qui se réunit pour la création, la modification ou la suppression de tous les diplômes de l'enseignement professionnel et technique du ministère de l'Éducation nationale. Elles sont rattachées à la Direction de l'Enseignement scolaire. Leur activité s'effectue sous la responsabilité du Secrétariat général des CPC qui veille au respect de quatre phases.

La phase d'opportunité a pour objectif de vérifier l'intérêt de créer ou de modifier un diplôme et s'interroge notamment sur les perspectives de recrutement, le contenu professionnel de la demande et les relations avec l'offre de diplômes existante. L'administration de l'Éducation nationale s'appuie sur des enquêtes statistiques et qualitatives (entretiens avec des employeurs, des salariés) pour valider ou non la demande, et définir une "cible professionnelle».

La construction du référentiel des activités professionnelles quant à elle vise à décrire les activités professionnelles prises en charge par le titulaire du diplôme quelques années après l'insertion professionnelle. La mise au point du référentiel de certification indique les conditions et les indicateurs d'évaluation des compétences détenues par le futur diplômé.

Enfin, la quatrième phase consiste à mettre au point les modalités de validation et les aspects réglementaires du diplôme. Au terme de ces phases, les projets de diplôme sont présentés pour avis à la CPC concernée.

Au nombre de vingt, les CPC reproduisent le système des relations professionnelles des principales branches économiques reconnues. Tant qu'il s'agissait de créer ou de réviser des diplômes spécialisés pour des branches bien identifiées, ce fonctionnement s'est avéré pertinent, même s'il lui a souvent été reproché d'apporter des réponses trop fragmentaires. Pour faire face au développement de la demande en diplômes transversaux, émanant d'abord de certains professionnels du secteur tertiaire, puis de la maintenance et de la production, il lui a été préféré une nouvelle organisation de la consultation qui accorde un rôle accru à des groupes de travail dont la composition est transversale à plusieurs branches.

fois la demande adressée par les professionnels ${ }^{3}$. Le rôle des représentants des salariés et des enseignants dans le processus de création du diplôme a été très faible. En effet, peu de débats ont eu lieu sur les conclusions de l'expertise effectuée pour vérifier l'opportunité de créer ce diplôme ${ }^{4}$. Un groupe de

\footnotetext{
${ }^{3}$ Afin de rendre évidente l'existence d'un nouveau métier et de prévenir toute critique de méconnaissance des emplois, l'UIMM (Union des industries métallurgiques et minières, aujourd'hui Union des industries et des métiers de la métallurgie) a pris le soin d'effectuer au préalable une étude auprès des entreprises de sept branches (industries métallurgiques et minières, imprimerie et industries graphiques, chimie et pharmacie, textile, habillement, plasturgie et industrie papetière).

${ }^{4}$ Pour ce diplôme, le Céreq a réalisé une étude d'opportunité qui recommandait la création de deux bacs pros distincts (Marquette, Mériot, Kirsch, 1994). Le premier répondait aux attentes des industries de transformation de la matière et de l'énergie, où il existe de fortes similarités en amont du processus de production. Le second, intitulé PSPA, correspondait à la logique défendue par l'UIMM pour les industries soumises au flux tendu où $\quad . . . / \ldots$
}

travail ad hoc a été créé5. Il transcendait les contours de la CPC de la métallurgie et réunissait des représentants de grandes entreprises (Péchiney, PSA, Renault, ...) de plusieurs branches (agro-alimentaire, cosmétiques, matériaux de construction, pâtes, papier, carton, textile).

Mais cette maitrise de l'Éducation nationale sur les décisions occulte l'accord de fond qui préexistait entre le Secrétariat général des CPC et les représentants de l'UIMM. Le travail d'élaboration d'une réponse éducative, sous la forme des diplômes trans-

\footnotetext{
.../...les procédés mécaniques sont fortement modélisés, mais dans lesquelles le rapport à la matière de l'opérateur est plus distant et où les spécificités du produit pèsent moins dans les activités. ${ }^{5} \mathrm{Ce}$ mode de consultation en dehors des branches et de leurs représentants caractérise aussi la création du CAP «Conduite de systèmes industriels » (CSI) en 2003. Il devient la norme pour les diplômes transversaux.
} 
versaux, à la demande sociale des professionnels en compétences transversales, s'en est trouvé facilité.

\section{La transversalité opposée à la spécialisation}

Le Secrétariat général des CPC et les représentants de l'UIMM partagent en effet les mêmes représentations et utilisent des catégories proches pour exprimer les évolutions du travail de production. De leur point de vue, le couple «diplômes transversaux - compétences transversales» est en voie de remplacer progressivement le couple "diplômes spécialisés spécialisation des fonctions » jusqu'alors en vigueur dans l'enseignement professionnel. Cette idée n'est certes pas nouvelle, puisqu'elle était déjà présente au milieu des années 60 au moment de la création du BEP (brevet d'études professionnelles) qui visait à élargir le CAP (certificat d'aptitudes professionnelle) et à sortir de la correspondance entre un diplôme et une fonction; elle s'appuie cependant sur un argumentaire renouvelé.

D’une part, la flexibilité des équipements automatisés et les nouvelles formes de coopération dans le travail nécessitent des personnels polyvalents, autonomes, réactifs et formés à des activités professionnelles anciennement divisées entre ouvriers et techniciens. La forme la plus aboutie de cette nouvelle figure professionnelle est celle de l'opérateur des industries de process (chimie, énergie, matériaux-céramiques...), et notamment de la sidérurgie (Monchatre, 2004). Mais dans le cas du bac pro PSPA, il s'agit de former ces personnel par l'école, alors que dans ces industries les entreprises ont fréquemment utilisé la formation professionnelle continue pour pourvoir ces postes avec des salariés expérimentés. D'autre part, la logique sectorielle ne correspond plus aux nouvelles réalités industrielles puisque, dans de nombreux secteurs, il importe plus de connaître les procédés de production que les spécificités des produits. Dans cet environnement, la professionnalité ne dépend plus de la connaissance des produits et de la maîtrise des procédés et des installations de production propres à chaque secteur, mais elle se définit par la capacité à intervenir sur des équipements automatisés où les opérations sont coordonnées et intégrées. Les activités ne sont plus centrées sur le produit, mais sur la conduite de l'outil qui le produit (Kirsch, 1997).
Toujours selon cet argumentaire, ces évolutions concomitantes imposent aux salariés des compétences transversales, communes à des fonctions et des branches diverses, qui doivent être transmises par l'école. La sphère éducative doit transmettre et évaluer non seulement des savoirs et des savoir-faire, mais aussi des compétences et des attitudes permettant de réaliser des activités dans des situations de travail différentes, ce qui consacre le passage de la logique des compétences de la sphère du travail à celle de l'école. Ainsi, les programmes et les contenus d'enseignement doivent être libellés sous la forme de découpages des savoirs ; il s'agit d'opérer un lien entre la mobilisation des ressources cognitives et des savoir-être, et leur transfert dans des situations et des actions inédites. Les contenus d'enseignement doivent favoriser le potentiel d'adaptation en dépassant les spécificités du poste et les connaissances afférentes. Ils doivent se dégager des particularités de l'environnement professionnel et des savoirs spécialisés, propres à l'approche sectorielle des diplômes (Marquette, 1997), pour accéder à des savoirs "pluritechniques » et occuper une fonction nécessitant «polyvalence et multi compétence » (CNDP, 1997).

Cette homogénéité des représentations va aider à la construction du référentiel des activités professionnelles et du référentiel de certification en termes de compétences. Dans ces documents s'opère ainsi une substitution de la notion de savoirs par celle de compétences, érigées en objectifs standardisés (Ropé, Tanguy, 1994). La généralisation de l'utilisation de la notion de compétences a rendu leur formulation suffisamment abstraite pour correspondre à des secteurs et des branches différents (Tanguy, 1991).

Plusieurs réserves peuvent être émises quant à ces représentations, même si la conception des diplômes est une opération délicate à effectuer, surtout quand il s'agit d'anticiper des transformations dans le monde professionnel. Tout d'abord, les rapports entre connaissances et action ne sont pas aussi aisés à comprendre que ne l'ont pensé les experts. Si certains psychologues cognitivistes et psychopédagogues ont démontré que la notion de compétences renvoie à l'action en situation et à la mobilisation des ressources cognitives pour agir, d'autres chercheurs en éducation s'interrogent sur la capacité à transférer 
ces compétences dans des situations inédites. Ils soulignent en outre que les connaissances ne doivent pas être considérées comme secondaires par rapport aux compétences transversales qui sont trop réductrices parce que centrées sur l'individu (Crahay, 2006). Il serait donc préférable de restaurer le découpage disciplinaire dans les programmes et de ne pas considérer, comme cela est le cas depuis l'apparition des référentiels de certification, que l'objectif du diplôme est d'atteindre les compétences caractéristiques d'une cible professionnelle.

Ensuite, comme pour d'autres diplômes, et notamment le bac pro « Productique mécanique » (Veneau, 1997), dans les référentiels de ce diplôme prédomine une certaine lecture évolutionniste du changement technique et organisationnel en tant que phénomène linéaire et homogène. Ces instruments éludent la diversité des choix productifs et des organisations sociales du travail. Ils présentent une vision uniforme de l'avenir des métiers de la production qui consiste à délaisser la réalisation des produits pour assurer le fonctionnement des équipements. Ces orientations ignorent les conclusions des recherches qui ont souligné la diversité des organisations du travail utilisant une même technologie (Eyraud, Maurice, D'Iribarne, Rychener, 1984) et la segmentation des équipes de production qui peut s'opérer par l'automatisation (Veneau et Mouy, 1995).

\section{Les intérêts particuliers des acteurs}

Des attentes plus spécifiques à chaque catégorie d'acteurs expliquent également leur engouement pour les diplômes transversaux. Derrière l'argumentaire économique et pédagogique se cachent, pour l'IUMM, des raisons plus politiques. Par l'intermédiaire des diplômes transversaux, les acteurs économiques cherchent à peser fortement sur la formation initiale, les diplômes représentant un élément de leur identité et de leur légitimité (Maillard, 2005a). La création d'un autre diplôme transversal - le BTS « Assistant de gestion PME-PMI » - est significative de cette tendance puisqu'il est directement issu de la volonté de la CGPME (Confédération générale des petites et moyennes entreprises) de disposer d'un diplôme pour accroître le potentiel d'attraction des petites et moyennes structures productives sur les jeunes et ainsi tenter de faire jeu égal avec les grandes entreprises (Quenson, 2001, op. cit.).

En définissant un nouveau métier, indifférent aux secteurs et aux produits, les professionnels souhaitent des recrutements conformes au nouveau modèle technique et organisationnel qu'ils encouragent. En effet, la métallurgie redoute des problèmes d'embauche si la spécialisation perdure et continue d'affilier les jeunes à des branches préférant ce mode de certification. La transversalité permet donc de disposer d'un avantage concurrentiel par rapport aux professionnels moins offensifs, ou moins écoutés par les experts. Enfin, pour emporter l'adhésion des experts du ministère qui sont à la recherche de moyens pour élever le niveau de formation, l'UIMM choisit un diplôme qui, formulé au niveau du bac pro, correspond à un niveau de qualification susceptible d'intéresser plusieurs branches.

Pour le ministère, les diplômes transversaux sont l'occasion de répondre aux critiques sur son immobilisme en clarifiant l'offre de diplômes en fonction des besoins affichés par les employeurs. Mais la réponse apportée à la demande des professionnels est aussi liée aux évolutions du système éducatif. Les diplômes transversaux permettent de lutter contre la fragmentation des diplômes et d'envisager un allègement du coût des examens. Alors que trop souvent la tendance des professionnels est d'encourager la multiplication des diplômes en fonction des emplois spécialisés, la demande de l'UIMM poursuit une logique contraire en proposant un diplôme pour plusieurs branches et plusieurs emplois. Cette même logique avait animé l'entreprise Renault lorsqu'elle avait demandé à l'Éducation nationale de créer le CAP «Exploitation d'installations industrielles» (EII) (Gorgeu et Mathieu, 2002). Ce modèle de diplôme peut aussi coïncider avec la volonté exprimée par certains jeunes au cours de l'enquête de retarder leur spécialisation après l'obtention du BEP. Il permet également d'espérer une réduction du chômage juvénile en élargissant le champ d'exercice des diplômes. Enfin, la transversalité est représentée comme une solution efficace pour préparer la mobilité professionnelle des salariés dans un monde industriel changeant où il est difficile de prévoir les emplois de demain. 


\section{LES STAGES NE COUVRENT PAS L'ENSEMBLE DU RÉFÉRENTIEL DE CERTIFICATION}

Dans tous les bacs pros, les périodes de formation en milieu professionnel comptent pour l'obtention du diplôme, conformément aux demandes des professionnels qui ont vu dans ces stages la condition d'une meilleure adaptation des compétences aux équipements et aux produits. Dans le cas du bac pro PSPA, la part de l'exercice professionnel dans l'alternance est encore plus significative que dans d'autres bacs pros, puisque la durée du stage est de dix-huit semaines contre seize en général. De plus, le coefficient attaché à cette période représente un tiers des épreuves du bac pro ${ }^{6}$.

Mais contrairement aux attentes, l'alternance ne facilite pas toujours l'acquisition des compétences. Les entreprises servent avant tout leur intérêt à court terme, soit en affectant les jeunes sur des postes assez éloignés du diplôme, soit en privilégiant la conduite de lignes, ce qui conduit à minorer les activités de préparation et d'amélioration (de la maintenance, de la production et de la qualité). En outre, la disparité des situations professionnelles selon les secteurs et les entreprises crée d'importants écarts de connaissances et de pratiques. Le système productif des entreprises connaît en effet différents états de développement qui obligent les établissements scolaires à organiser l'acquisition des savoirs sur leurs propres équipements. Cette situation est pour le moins paradoxale pour un diplôme censé faciliter la transmission des connaissances professionnelles par un régime d'alternance accrue.

\section{L'exécution prime sur l'amélioration du système productif}

Depuis la création du diplôme, les chefs de travaux ${ }^{7}$ rencontrent des difficultés pour trouver des entreprises correspondant aux objectifs du diplôme. Outre

\footnotetext{
${ }^{6}$ L'examen comprend aussi des épreuves scientifiques et techniques, de technologie, langue vivante, français, histoire-géographie, éducation artistique/arts appliqués et éducation physique et sportive.

${ }^{7}$ Placé sous l'autorité du proviseur de lycée technologique ou professionnel et dans le cadre du projet d'établissement, le chef de travaux joue un rôle essentiellement d'organisateur et de conseiller. . .../...
}

le fait que dans bien des structures productives, une grande partie des équipements n'est pas encore automatisée, quand des postes de conducteurs de ligne existent, les responsables hésitent à les confier à des jeunes en cours d'études. De plus, les tuteurs, euxmêmes généralement conducteurs de ligne, ne bénéficient pas de décharges horaires ou de production, tant les effectifs sont aujourd'hui réduits dans les entreprises, ce qui limite leur disponibilité pour transmettre et évaluer les connaissances des jeunes.

Deux régimes d'alternance sont observés. Le premier concerne l'utilisation des jeunes en début et en fin de ligne dans les activités d'approvisionnement et d'empaquetage. Cette spécialisation précoce dans l'amont et l'aval du processus de production réduit leur champ d'intervention. Elle rend impossible la réalisation des activités de conduite de lignes prévues par le référentiel et nuit à toute augmentation progressive des connaissances dans ce domaine d'activité, pourtant central pour l'obtention du diplôme. Cette situation découle aussi de la tendance des équipes pédagogiques à privilégier le placement en entreprise, au lieu de vérifier l'entière conformité du lieu de stage avec les objectifs du diplôme (Farvaque, 2009).

Un second régime d'alternance concerne plutôt des entreprises, anciennement industries de maind'œuvre, connaissant une phase soutenue de modernisation technique. De taille importante (autour de 200 salariés), appartenant aux secteurs de l'agroalimentaire, de la mécanique et de la parachimie, ces structures productives cherchent à diminuer leurs effectifs de production; pour ce faire, elles anticipent les futurs recrutements par l'accueil de jeunes capables de produire plus vite et, au besoin, d'entretenir les machines afin de prévenir toute interruption. Ainsi la tentation est grande, chez certains employeurs, de brûler les étapes de la préparation au diplôme pour utiliser les jeunes sur des postes d'opérateurs. Ces stratégies induisent un apport limité en connaissances professionnelles par rapport à l'étendue des activités devant être couvertes. La

\footnotetext{
.../...Il participe au choix des équipements, délivre des informations sur les évolutions des métiers et des technologies en entreprise, aide à la formation des enseignants et veille à la conformité des locaux et des matériels pédagogiques.
} 
participation aux opérations de maintenance préventive et corrective et à l'analyse des résultats produits par les outils de la qualité est ainsi minorée au profit de la conduite de lignes. Il en va de même des activités d'animation et de coordination des équipes de production. Si l'identification des informations relatives à la production est une activité effectuée de manière assez systématique, ce qui est congruent avec le choix des entreprises de faire des jeunes nouvellement formés les agents d'une modernisation accélérée des techniques, les activités de formation des équipes et d'amélioration de la production par la participation à des groupes de progrès ou à des cercles de qualité sont la plupart du temps délaissées. La division du travail qui perdure entre techniciens et personnels de production explique que les jeunes soient exclus de ces activités qui sont estimées périphériques à la production proprement dite. De plus, conformément aux principes de la production en flux tendus ${ }^{8}$, celles-ci se répètent à intervalles réguliers selon des procédures très normalisées connues des seuls personnels techniques expérimentés (préparation du poste de travail et des données de production, interprétation des dérives et des dysfonctionnements).

Le secteur industriel explique aussi pourquoi certains stagiaires sont insuffisamment formés et évalués dans certaines activités. Dans l'agro-alimentaire par exemple, la conduite en mode dégradé ${ }^{9}$ ne fait pas partie des activités de production. En effet, compte tenu de l'importance attachée à l'hygiène et à la qualité des produits, de multiples indicateurs révèlent les dysfonctionnements bien avant la mise en production.

Ainsi, ces conditions aboutissent à une certaine désillusion de la part des jeunes. L'écart perçu entre l'emploi occupé temporairement et leurs aspirations initiales à accéder à un poste de technicien nourrit leur déception (Moreau, 2008). Leur première expérience du travail vient apporter un démenti aux espé-

\footnotetext{
${ }^{8}$ Le flux tendu est une méthode de production industrielle issue du toyotisme qui consiste à réduire au minimum les temps de passage des produits au cours des différentes étapes de leur élaboration. Il s'accompagne d'une diminution des stocks et des en-cours de fabrication.

${ }^{9}$ Ce type de conduite s'effectue quand des dégradations sont observées sur les produits ou les installations. Il nécessite l'application de procédures spécifiques pour retrouver une fabrication stabilisée.
}

rances qu'ils avaient fondées dans le diplôme comme moyen d'échapper à la condition ouvrière.

Contrairement aux prévisions des concepteurs du diplôme, les évolutions techniques, quand elles sont avérées, ne s'accompagnent pas toujours d'une modernisation sociale de l'organisation du travail ; cela réduit les marges de manœuvre des stagiaires qui doivent se fondre dans des organisations préexistantes plus marquées par la rigidité que par la recomposition des frontières entre fonctions et métiers. Ils doivent de fait accepter une affectation sur des postes qui les éloignent de la préparation au diplôme.

\section{Une mauvaise répartition des tâches entre instances de formation et entreprises}

Le décalage entre le contenu de la période de formation en entreprise et les objectifs des référentiels explique pourquoi les établissements sont amenés à compléter la formation sur leurs propres équipements. Dans le cas du premier régime d'alternance, les établissements ont dû se munir de lignes automatisées pour compenser le manque de pratique des jeunes sur ces équipements. Ceci a été possible, au bout de quelques années, grâce à des investissements consentis par certains conseils régionaux, voire à la suite de dons émanant d'entreprises.

Quant au second régime d'alternance, la conduite dans des conditions de production normale et la maintenance de premier niveau font généralement l'objet d'une évaluation en entreprise. En revanche, la conduite en mode dégradé, l'amélioration de la qualité et de la maintenance, et l'encadrement des équipes de travail sont fréquemment délaissés. Pour remédier à ces manques, les enseignants sont amenés à créer des situations de production fictives. Certains établissements recourent ainsi à des élèves des classes inférieures qu'ils assimilent à des opérateurs pour évaluer la manière dont les stagiaires effectuent le conseil technique des opérateurs affectés sur les lignes. La mesure des capacités des jeunes à travailler en conduite dégradée s'effectue aussi en dehors des conditions réelles de la production.

La méthodologie des référentiels ( $c f$. encadré 2), qui suppose une mise en forme plus didactique et abstraite que professionnelle des attentes de la 
formation et des résultats de la certification, explique aussi certainement pourquoi nombre d'entreprises délaissent l'évaluation d'une grande part des activités professionnelles. Discernant difficilement comment elles vont donner un contenu concret à l'acquisition des capacités et des compétences dénommées par les référentiels, elles se tournent vers les enseignants qui sont peut-être, de leur point de vue, les acteurs les plus à même de rendre intelligible le registre des référentiels. Mais parfois cet exercice de traduction ne suffit pas à rendre possible l'évaluation des jeunes par les entreprises. Les équipes pédagogiques des établissements doivent se substituer aux employeurs et inventer des modes d'évaluation assez éloignés des conditions concrètes du travail.

La période de formation en entreprise s'avère donc assez conforme à une vision utilitariste et de court terme du diplôme qui se limite bien souvent à préparer des conducteurs de lignes dans la perspective d'un simple remplacement des ouvriers qualifiés. Les entreprises profitent alors pleinement de l'autonomie et de l'efficacité des jeunes qui sont assimilés à des personnels à part entière. Cette interprétation minimaliste des connaissances transmises par le milieu professionnel préfigure, pour une large part, les conditions d'insertion.

\section{PERTINENCE DE}

\section{LA TRANSVERSALITÉ : SECTEUR, DEGRÉ D'AUTOMATISATION ET DIVISION DU TRAVAIL}

L'insertion met à l'épreuve les écarts entre la formation et les activités professionnelles. De nombreux espaces existent entre le modèle et les activités concrètes. La vision d'un mode de rationalisation unique, représentée par le diplôme, se heurte aux choix organisationnels et sociaux des entreprises. En effet, les relations entre les diplômes et les emplois ne suivent pas une voie linéaire entre le titre scolaire détenu et la place occupée par les jeunes. Elles s'établissent au terme d'une chaîne de médiations complexes.

En la matière, trois caractéristiques interagissent pour décider de l'insertion: le secteur industriel et les caractéristiques des produits, le degré de l'automatisation et les types d'activité qui en découlent, et les coupures fonctionnelles établies par la division du travail. La combinaison de ces caractéristiques infirme souvent les prévisions d'emploi initialement déterminées dans la phase de création du diplôme.

\section{Le secteur industriel détermine le contenu du travail}

Le contenu du travail se rapproche le plus des objectifs du diplôme dans les secteurs en aval de la filière industrielle intervenant à l'issue des différentes phases de transformation de la matière, où les produits fabriqués conservent la même forme une fois effectuée la commercialisation (automobile, cosmétiques, imprimerie, informatique, mécanique, routage...). Les activités principales consistent à conduire un segment de production automatisée comprenant plusieurs niveaux de commande et de gestion, et à optimiser les procédés dans l'approvisionnement, l'assemblage et le conditionnement des produits. Le rythme de production est élevé, les changements de format et de série sont nombreux, en raison de la diversité des produits et de la variabilité des commandes. Les contrôles sont fréquents et les incidents doivent être rapidement surmontés par des diagnostics pertinents et des interventions en cours de fabrication pour remédier aux dysfonctionnements et apporter des propositions d'amélioration. Les conducteurs intègrent une partie des fonctions non directement productives, telles que la maintenance des appareillages (graissage, vérification de l'usure des pièces, nettoyage...), le contrôle, la qualité des produits, ce qui nécessite une bonne compréhension des phénomènes techniques.

Les entreprises constatent une nette élévation des connaissances des jeunes par rapport aux salariés plus anciens. Pour autant, la reconnaissance du diplôme est faible. De ce point de vue, la transversalité ne représente pas un avantage par rapport à la spécialisation. Les titulaires du diplôme occupent un rang identique à celui des titulaires de diplômes plus spécialisés (Combes et Quenson, 2001; Eckert, 1999). Le niveau professionnel qui leur est attribué est toujours celui d'ouvrier qualifié, alors que les concepteurs du diplôme prévoyaient l'accès aux fonctions techniques des ateliers. 
Dans les secteurs situés en amont de la filière industrielle, qui rassemblent des industries transformant directement la matière (agro-alimentaire, chimie, papiers et carton, plasturgie), le diplôme est encore moins reconnu. Les jeunes ne travaillent pas là où les matières premières subissent des transformations importantes. C'est le cas par exemple des activités de stérilisation du lait dans l'agro-alimentaire, de production de films plastiques et de plastiques ABS (thermoplastique) dans la plasturgie et de caoutchouc dans la chimie. La transversalité s'avère moins pertinente dans le cas où le conducteur de ligne doit gérer des systèmes techniques complexes, appréhender de manière directe les matériaux, et maîtriser les procédés de transformation physicochimiques de la matière, effectuer des prélèvements et les interpréter par pesées, contrôles visuels et prises de température, c'est-à-dire dans les activités de type "process ", au sens utilisé dans les industries de flux de type chimie de base, sidérurgie ou encore dans une partie des activités de l'agroalimentaire. Dans ces secteurs industriels où une partie de la production se situe au niveau des procédés primaires de transformation, avant de prétendre à un poste de conducteur, il est nécessaire de posséder des connaissances sur le produit et ses réactions physico-chimiques; ces connaissances étant attestées par des diplômes spécialisés, des formations continues spécifiques et des années d'expérience. En conséquence, les jeunes sont cantonnés à des emplois peu qualifiés dans l'approvisionnement et le conditionnement des produits, assortis de faibles perspectives de mobilité.

La situation de ces entreprises pose la question du degré de spécialisation des connaissances sur les procédés et les produits nécessaires à l'expertise, à laquelle la transversalité ne propose pas de réponses appropriées. Pour ces différentes raisons, les diplômes spécialisés continuent d'apporter davantage de garantie aux yeux des professionnels, surtout quand les traditions de gestion du personnel n'ont pas intégré la possibilité de spécialiser les salariés de production par la voie de la formation professionnelle continue. Cette réaction est aussi plus fréquente dans les petites et moyennes entreprises, plus dispersées du point de vue sectoriel et sur le territoire, pour qui la formation scolaire représente la ressource la moins coûteuse, parce qu'elle est prise en charge par la collectivité nationale.

\section{Une automatisation inégale}

Globalement, l'automatisation existe dans les entreprises qui ont remplacé leurs machines traditionnelles par des lignes organisées en îlots, totalement contrôlées par informatique et supervisées par des automates programmables permettant une production en flux tendus. Parmi tous les segments de production, le conditionnement représente l'activité où elle est la plus aboutie. Mais ce niveau de sophistication technologique et cette intensité capitalistique ne caractérisent pas l'ensemble des entreprises. L'automatisation ne se résume pas à une alternative unique derrière laquelle se rangeraient en ordre bien pensé toutes les organisations productives. Une bonne moitié des entreprises automatisent leur "process » de manière très progressive. Elles conservent encore des machines manuelles dédiées à des séries plus courtes où l'automatisation s'avère peu rentable, si bien que plusieurs technologies se côtoient fréquemment dans les ateliers.

Par ailleurs, l'automatisation des équipements de production ne suit pas une évolution linéaire. L'exemple d'une entreprise japonaise produisant $\mathrm{du}$ matériel bureautique est, de ce point de vue, éloquent. Après avoir automatisé la production pour augmenter la productivité du travail et répondre à la croissance du marché européen, elle privilégie désormais la diversité et la qualité pour faire face à un ralentissement des ventes. Cette évolution nécessite une nouvelle stratégie industrielle et commerciale. Les produits standardisés sont progressivement remplacés par une gamme de produits diversifiés correspondant davantage aux demandes des clients. Ces changements concernent directement la production où les îlots de production flexibles constitués de petits robots peu consommateurs d'énergie - dont le fonctionnement est simple et le coût moindreremplacent les lignes entièrement automatisées, moins flexibles et plus coûteuses. Après avoir remplacé l'homme par des robots, cette usine consacre le retour de l'homme assisté par des robots.

Ce fabricant de photocopieurs reconnaît plusieurs avantages à ce système inspiré pour partie du 
«toyotisme ». L'investissement en machines est minime. Les convoyeurs ont disparu, puisque les opérateurs effectuent manuellement le transit des pièces d'un poste de travail à l'autre. Il est aussi possible de changer plus facilement de production. Selon les impératifs, les personnels peuvent être affectés d'un atelier à l'autre. Les machines étant simples, les ouvriers de maintenance ne sont plus indispensables. La rentabilité à court terme s'en trouve accrue. En revanche, le travail des opérateurs, recrutés depuis plusieurs années au niveau du bac pro, a considérablement perdu en intérêt. Les tâches à effectuer sont encore plus simples et plus répétitives que sur les anciennes lignes automatisées. Cette évolution du travail pourrait se traduire par une démobilisation des opérateurs les plus qualifiés.

\section{Une recomposition partielle de la division du travail}

Comme dans de nombreux bac pro de la production, les traditions de recrutement des personnels d'atelier et l'état de la division du travail représentent des obstacles fréquents à la reconnaissance du travail des jeunes nouvellement titulaires du diplôme (Veneau et Mouy, 1995, op. cit.; Eckert, 1999, op. cit.). La pratique qui consiste à penser qu'à toute nouvelle fonction dans l'entreprise doit correspondre un diplôme spécifique et un niveau de formation adéquat fait fi des usages en matière de mobilités professionnelles ; ces dernières permettant bien souvent aux organisations productives de pourvoir les emplois grâce à des salariés expérimentés ayant suivi divers cheminements professionnels dans et hors de l'entreprise et du secteur. Elle ignore aussi l'extrême diversité qui existe entre les salariés occupant un même poste de travail en matière de spécialités, de niveaux de formation et d'expériences professionnelles.

Ainsi, la plupart des entreprises enquêtées ont recruté pendant longtemps des personnels non diplômés issus du salariat agricole, du petit commerce, de l'artisanat ou de l'industrie, ou des personnels diplômés du CAP/ BEP dans diverses spécialités de la production, pour les former à des postes de conducteurs, situés audessus des ouvriers non qualifiés dans l'échelle des responsabilités. Elles ont perçu l'arrivée de jeunes diplômés comme une opportunité pour remplacer une partie du personnel expérimenté mais vieillissant.
Pour autant, cette hausse du niveau de recrutement ne s'accompagne pas d'une élévation du niveau des emplois plusieurs années après la sortie du système scolaire. La structure des qualifications reste bien souvent inchangée. Cette situation peut s'expliquer par le recrutement de nombreux diplômés dans des industries de "process » qui ne sont pas réputées pour respecter une correspondance forte entre les spécialités de formation et les types d'emplois.

Ces conditions de recrutement sont préjudiciables aux jeunes dans la mesure où la reconnaissance du diplôme dépend largement de l'état de la division sociale du travail. L'efficacité de la transversalité suppose des organisations du travail reconfigurées où les frontières entre les fonctions de production ont bougé, notamment en faveur des salariés affectés sur des lignes. Or, dans la plupart des entreprises, l'automatisation des équipements de production ne s'accompagne pas d'une refonte d'ensemble des organisations du travail.

En conséquence, une grande partie des activités professionnelles prévues par le référentiel d'emploi du diplôme échappe aux jeunes, le décloisonnement des fonctions étant très partiellement réalisé. Comme dans les stages, les activités les plus fréquemment délaissées sont celles qui ne sont pas directement liées à la production. Elles incombent encore aux personnels techniques qui bénéficient d'une position dominante dans les ateliers.

Néanmoins, dans une minorité d'entreprises situées en aval de la filière industrielle et dans les secteurs d'approvisionnement et de conditionnement des industries situées en amont, l'emploi de conducteurs de ligne peut déboucher sur un emploi de pilote reconnu dans les grilles de classification au niveau des premiers échelons de la catégorie des techniciens, mais seulement après cinq années d'expérience en moyenne et une sélection drastique. Dès lors, il ne s'agit pas d'un débouché naturel pour la majeure partie des conducteurs. Le développement de ce niveau hiérarchique correspond à la constitution d'un nouvel encadrement de proximité. Cette pratique montre que les entreprises éprouvent le besoin d'assister les conducteurs par un premier encadrement qui fait le lien entre l'atelier et les services techniques. Elle vient contredire le discours, assez consensuel dans les milieux patronaux, sur 
l'évolution du travail ouvrier vers davantage d'autonomie et de polyvalence, conduisant à la quasidisparition de l'encadrement de proximité.

Puisque les entreprises disposent de conducteurs de lignes d'une part, de pilotes assurant la coordination des équipes de production d'autre part, et enfin de techniciens affectés aux services de qualité et de maintenance, elles ne représentent pas un terrain propice au transfert des savoirs acquis en formation dans le champ professionnel, du moins dans les premières années suivant l'obtention du diplôme. Le travail confié au diplômé n'existe pas tel qu'il est défini dans le référentiel d'emploi, puisque des clivages importants subsistent entre la fabrication proprement dite et les activités techniques. Du reste, le même problème a été mis en lumière pour un autre diplôme transversal de la production: le CAP «Exploitation d'installations industrielles » (EII) (Coutelier, 2000).

Deux cas de figure apparaissent donc en matière de reconnaissance du diplôme par le monde du travail. Dans les entreprises intervenant en aval du processus de production industrielle, dont les équipements sont automatisés, les jeunes occupent des emplois dans la fabrication des produits finis, où ils remplacent les ouvriers expérimentés et sont classés dans la catégorie des ouvriers. La transversalité est largement sous-utilisée puisqu'une grande partie des connaissances et des savoir-faire ne sert pas les entreprises. Seule une minorité de jeunes pourra accéder, mais sans certitude aucune, à des postes de responsables de proximité, situés dans les premiers échelons de la catégorie des techniciens.

Dans les entreprises situées en amont de la filière industrielle, les jeunes occupent des emplois de conducteurs dans les seuls secteurs d'approvisionnement et de conditionnement. Dans ces secteurs, les employeurs se réfèrent encore à des critères d'évaluation traditionnels qui accordent une grande importance à l'idée de «métier». La spécialisation des diplômes reste très appréciée, parce qu'elle est synonyme de capacités techniques ajustées aux équipements et aux procédés de production, et est associée à une connaissance fine des produits. Là encore, l'utilisation de la transversalité est faible et les perspectives de carrière réduites. Seule une minorité pourra se voir proposer des mobilités vers des postes de responsable de premier niveau. Mais certaines mobilités sont plus illusoires, puisque les connaissances initiales doivent être complétées par des savoirs sur les produits et leurs réactions aux transformations. Seule l'expérience, doublée de formations professionnelles complémentaires, permettra à certains d'atteindre ces postes, à condition toutefois que les entreprises ne leur préfèrent pas des diplômés déjà spécialisés, dont le coût de formation est moindre.

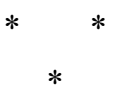

$\mathrm{Au}$ terme de cette analyse, il convient de dresser quelques constats sur la pertinence du modèle de certification du bac pro PSPA (« pilotage de système de production automatisé ») du point de vue de sa conception, puis de son utilisation par le marché du travail.

L'analyse de l'élaboration et des conditions de matérialisation du diplôme montre que les catégories de questionnement utilisées par les acteurs, qui ont opposé la transversalité à la spécialisation des diplômes et la polyvalence à la spécialisation des postes et des fonctions, ont été mal posées dès le départ. L'Éducation nationale et les représentants des professionnels ont d'abord mésestimé la tendance des secteurs industriels et des entreprises à définir l'organisation du travail et le contenu des emplois de production de façon indépendante, dans une logique qui leur est spécifique, et non dans une logique interprofessionnelle favorable à la transversalité du diplôme. Ils ont aussi considéré que le système productif et les techniques suivent une évolution linéaire.

Or la réalité est plus ambiguë. La distinction entre transversalité et spécialisation et entre polyvalence et spécialisation des métiers est bien plus aléatoire que les prévisions ne l'annonçaient. Les contradictions sont constantes. Elles demeurent dans le cas de ce diplôme. Ainsi, la polyvalence, qui est l'objectif apparemment recherché par la plupart des entreprises, a trait en réalité plus fréquemment au poste de travail immédiat qu'à l'approfondissement d'une professionnalité en devenir qui concernerait la participation aux activités connexes de production, 
comme l'avait pensé initialement les concepteurs du diplôme en prenant le parti, peut-être, d'une trop grande anticipation de la réalité. Enfin, une autre erreur de diagnostic s'inscrit dans une vision quelque peu sommaire des rapports entre connaissance et action dans les activités de process, qui a conduit à minorer leur complexité.

Sur le marché du travail, l'exemple de ce diplôme illustre la tendance de certains professionnels à reporter sur la collectivité nationale la formation de leurs futurs personnels, alors que d'autres alternatives sont possibles. On peut en effet estimer que nombre d'emplois de conducteurs - finalement la cible professionnelle la plus généralement rencontrée au cours de l'enquête - pourraient être pourvus par des personnels expérimentés et formés de manière progressive, pour qui cette mobilité représenterait une reconnaissance de valeur professionnelle et un déblocage de carrière. Dans l'absolu, la formation professionnelle continue permet d'ajuster, de former et de compléter les connaissances et les compétences des personnels en place. Mais il est vrai qu'elle nécessite a minima une réflexion interne à l'entreprise sur l'évolution des métiers, des organisations du travail et sur les finalités à atteindre. De plus, pour des entreprises qui ont pratiqué pendant longtemps une politique sélective d'accès à la formation privilégiant les catégories de personnel les plus qualifiées, le développement d'une politique de formation orientée vers les conducteurs en poste nécessite un changement des habitudes et des comportements qui ne pourra se réaliser qu'après quelques années d'efforts constants.

Le recours aux diplômes permet de résoudre une grande partie de ces problèmes. Outre le fait qu'ils reportent sur d'autres acteurs que l'entreprise les modalités et l'organisation de la formation, les diplômes présentent l'avantage de garantir les savoirs et les compétences des jeunes, la rapidité de leur adaptation aux postes de travail et leur intégration dans les équipes. Or, malgré ces avantages, la majeure partie des entreprises ne reconnaît pas les qualités des jeunes au niveau attendu par les concepteurs du diplôme, si bien que l'on peut noter de leur part une propension à profiter de l'effet d'aubaine que constitue actuellement le diplôme par rapport à tout autre mode de formation.

Un diplôme ne formant pas pour un emploi spécifique, mais pour une carrière, les modalités d'entrée dans la vie active posent ainsi des interrogations sur la capacité des jeunes à intégrer, après quelques années, des emplois plus qualifiés correspondant davantage à leur formation initiale. Les cheminements et les espaces de mobilité qui suivront dépendront fortement des modalités de reconnaissance de l'expérience, des possibilités effectives de promotion et du rôle attribué à la formation professionnelle. En la matière, de nouvelles opportunités existeront certainement, mais elles dépendront aussi des secteurs, de la taille des entreprises, des choix organisationnels et des politiques de gestion du personnel. 


\section{Bibliographie}

Centre national de documentation pédagogique (1997), Référentiel du baccalauréat professionnel «Pilotage de systèmes de production automatisée ».

Combes M.-C., Quenson E. (2001), «Évaluation des baccalauréats professionnels de la productique mécanique », MEN, CPC/Documents, $\mathrm{n}^{\circ} 6$.

Couppié T., Giret J.-F., Lopez A. (2005), «Des formations initiales aux premiers emplois: une correspondance mal assurée », in Giret J.-F., Lopez A., Rose J. (Ed.), Des formations pour quels emplois ?, La Découverte, Paris, pp. 79-96.

Coutellier F. (2000), « Le CAP exploitation d'installations industrielles. Son développement en formation initiale. La situation des jeunes en entreprise », MEN, CPC/Documents, $\mathrm{n}^{\circ} 7$.

Crahay M. (2006), « Dangers, incertitudes et incomplétude de la logique des compétences en éducation ", Revue française de pédagogie, $\mathrm{n}^{\circ} 154$, pp. $97-110$.

Eckert H. (1999), « L'émergence d'un ouvrier bachelier. Les bac pro entre déclassement et recomposition de la catégorie des ouvriers qualifiés ", Revue française de sociologie, $\mathrm{n}^{\circ} \mathrm{L}-2$, pp. 227-253.

Eyraud F., Maurice M., D’Iribarne A., Rychener F. (1984), «Développement des qualifications et apprentissage par l'entreprise des nouvelles technologies: le cas des machines-outils à commande numérique », Sociologie du travail, $n^{\circ} 4$.

Farvaque N. (2009), « Discrimination dans l'accès au stage : du ressenti des élèves à l'intervention des enseignements », Formation Emploi n ${ }^{\circ} 105$.

Fourcade B. (1997), «La création des premiers baccalauréats professionnels de la métallurgie: maintenance, productique, électrotechnique », in Möbus M., Verdier E. (Ed.), Les diplômes professionnels en Allemagne et en France. Conception et jeux d'acteurs, L'Harmattan, Paris, pp. 105-128.
Gorgeu A., Mathieu R. (2002), « Le CAP Exploitation d'installations industrielles : une seconde chance pour les non-diplômés ? », Formation Emploi, $\mathrm{n}^{\circ}$ 80, pp. 53-69.

Kirsch E. (1997), «Diplôme transversal, diplôme pour quelles entreprises ? », in Möbus M., Verdier E., in Les diplômes professionnels en Allemagne et en France. Conception et jeux d'acteurs, pp. 209-228.

Maillard F. (2005a), Pour un regard sociologique sur la formation et les diplômes professionnels, Mémoire pour l'habilitation à diriger des recherches, Faculté de philosophie, sciences humaines et sociales, université de Picardie.

Maillard F. (2005b), «L'offre des diplômes professionnels de l'Éducation nationale en France : injonctions contradictoires ", Cahiers de la recherche sur l'éducation et les savoirs, ${ }^{\circ} 4$, pp. 35-60.

Marquette C. (1997), «Spécialisation et diplômes professionnels : le cas du baccalauréat professionnel Process ", in Möbus M., Verdier E., in Les diplômes professionnels en Allemagne et en France. Conception et jeux d'acteurs, pp. 191-208.

Marquette C., Mériot S.-A., Kirsch E. (1994), «Création d'un baccalauréat professionnel productique dans les industries de process », MEN, $C P C /$ Documents, $\mathrm{n}^{\circ} 3$.

Möbus M., Verdier E. (Ed.) (1998), Les diplômes professionnels en Allemagne et en France. Conception et jeux d'acteurs, L'Harmattan, Paris.

Monchatre S. (2004), «De l'ouvrier à l'opérateur : chronique d'une conversion", Revue française de sociologie, $\mathrm{n}^{\circ}$ 41-I, pp. 69-102.

Moreau G. (2008), «Apprentissage : une singulière métamorphose», Formation Emploi, $\mathrm{n}^{\circ}$ 101, pp. 119-133.

Ourtau M. (1995), «Étude d'opportunité et recours à l'expertise dans les processus de création des diplômes », Formation Emploi, n 52, pp. 67-85. 
Pillet M. (1995), «La création du baccalauréat professionnel. Les processus de décision », Formation Emploi, $\mathrm{n}^{\circ}$ 52, pp. 87-99.

Quenson E. (2001), «Évaluation du BTS assistant de gestion PME-PMI », MEN, CPC/Documents, $\mathrm{n}^{\circ} 2$.

Quenson E. (2004), « Un nouveau profil de diplôme pour une nouvelle figure professionnelle ? Le baccalauréat professionnel pilotage de système de production automatisée, un diplôme transversal », MEN, CPC/Documents, $\mathrm{n}^{\circ} 8$.

Quenson E. (2008), «Les diplômes transversaux améliorent-ils la correspondance entre la formation et l'emploi ? », in Maillard F (Dir.), Des diplômes aux certifications professionnelles? Nouvelles normes et nouveaux enjeux, Presses Universitaires de Rennes, Rennes, coll. « Des sociétés».
Ropé F., Tanguy L. (1994), Savoirs et compétences. De l'usage de ces notions dans l'école et l'entreprise, L'Harmattan, Paris.

Tanguy L. (1991), Quelle formation pour les ouvriers et les employés en France?, Rapport au Secrétariat d'État à l'Enseignement technique, La Documentation française.

Veneau P. (1997), «Construction de référentiels et analyse prospective du travail: l'exemple de l'usinage mécanique », in Möbus M., Verdier E., in Les diplômes professionnels en Allemagne et en France. Conception et jeux d'acteurs, pp. 163-173.

Veneau P., Mouy P. (1995), «Des objectifs à la réalité. Les bacheliers professionnels industriels », Formation Emploi, n ${ }^{\circ}$ 49, pp. 91-103.

Verdier E. (1995), «Négocier et construire des diplômes », Formation Emploi, n ${ }^{\circ}$ 52, pp. 3-10.

\section{Résumé}

\section{Les diplômes transversaux peinent à s'imposer sur le marché du travail Emmanuel Quenson}

Contrairement aux diplômes spécialisés, représentatifs d'une période où les diplômes de l'enseignement professionnel permettaient d'accéder à des qualifications déterminées dans des branches spécifiques, les diplômes transversaux sont censés s'ajuster à la flexibilité des organisations et à la recomposition des métiers dans plusieurs branches. Pourtant, l'exemple d'un bac pro de la production montre que la transversalité peine à s'imposer sur le marché du travail. Ce constat conduit à s'interroger sur la difficulté à élaborer de nouveaux modèles de diplômes qui doivent anticiper les transformations des entreprises.

Mots clés

Baccalauréat professionnel, technicien de production, relation école-entreprise, CPC - commission professionnelle consultative, compétence transversale

Journal of Economic Literature: J 23, J 31 